\title{
Multi-modal image registration: matching MRI with histology
}

\author{
Lejla Alic $^{\text {ab }}$, Joost C. Haeck ${ }^{\mathrm{b}}$, Stefan Klein ${ }^{\mathrm{ab}}$, Karin Bol ${ }^{\mathrm{ab}}$, Sandra T. Van Tiel ${ }^{\mathrm{b}}$, \\ Piotr A. Wielepolski ${ }^{b}$, Magda Bijster ${ }^{\mathrm{c}}$, Wiro J. Niessen ${ }^{\text {abd }}{ }^{\text {, Monique Bernsen }}{ }^{\mathrm{b}}$, Jifke F Veenland ${ }^{\mathrm{ab}}$, \\ Marion de Jong ${ }^{\mathrm{c}}$
}

${ }^{a}$ BIGR - Biomedical Imaging Group Rotterdam, Departments of Radiology ${ }^{\mathrm{b}}$ and Nuclear Medicine ${ }^{\mathrm{c}}$, Erasmus MC, Rotterdam, P.O. Box 2040, 3000 CA Rotterdam, The Netherlands

${ }^{\mathrm{d}}$ Faculty of Applied Sciences, Delft University of Technology, Delft, The Netherlands

\begin{abstract}
Spatial correspondence between histology and multi sequence MRI can provide information about the capabilities of non-invasive imaging to characterize cancerous tissue. However, shrinkage and deformation occurring during the excision of the tumor and the histological processing complicate the co registration of MR images with histological sections. This work proposes a methodology to establish a detailed 3D relation between histology sections and in vivo MRI tumor data. The key features of the methodology are a very dense histological sampling (up to 100 histology slices per tumor), mutual information based non-rigid B-spline registration, the utilization of the whole 3D data sets, and the exploitation of an intermediate ex vivo MRI.

In this proof of concept paper, the methodology was applied to one tumor. We found that, after registration, the visual alignment of tumor borders and internal structures was fairly accurate. Utilizing the intermediate ex vivo MRI, it was possible to account for changes caused by the excision of the tumor: we observed a tumor expansion of $20 \%$. Also the effects of fixation, dehydration and histological sectioning could be determined: $26 \%$ shrinkage of the tumor was found. The annotation of viable tissue, performed in histology and transformed to the in vivo MRI, matched clearly with high intensity regions in MRI. With this methodology, histological annotation can be directly related to the corresponding in vivo MRI. This is a vital step for the evaluation of the feasibility of multi-spectral MRI to depict histological groundtruth.
\end{abstract}

Keywords: multi-modal registration, MRI, histology, tumor, tissue characterization

\section{INTRODUCTION}

Multi-modal MR imaging is often used for non-invasive tumor characterization. To assess what information can be derived from the different MRI sequences, determining the spatial relation between MR images and ground truth, e.g. histology, would be highly beneficial. The accuracy with which this registration can be achieved directly affects this analysis. Although the registration among in vivo imaging modalities is well established, accurate registration involving histology remains difficult and has been explored during the last decade. The early non-rigid registration approaches consist of registering MRI images using surface matching to cryosections ${ }^{1}$, or volume marching to histological sections ${ }^{2}$. At present, results that have been reported have been limited to global matching of MRI volume to small numbers of histological sections. To create histological images, tumor tissue undergoes excision, fixing by formalin consisting in a precipitation of the tissue proteins followed by dehydration. An important side effect of the precipitation is a decreasing ability of proteins to keep the water resulting in a deformation of the tissues and tearing artefacts. All these actions inevitably change the tumor morphology and thereby complicate the registration of in vivo MRI to histological sections.

Simple visual colocalization based on local tissue features ${ }^{3}$ does not take into account the 3D deformations of the histology slices and is prone to observer bias. In more advanced experimental ${ }^{4}$ and clinical studies ${ }^{5-7}$, point based registration derived from manual placement of control points along the boundary has been used. The limited number (usually up to 7) of control points restricts thereby the capability of this method to model complex elastic deformations due to tissue processing.

Medical Imaging 2010: Biomedical Applications in Molecular, Structural, and Functional Imaging,

edited by Robert C. Molthen, John B. Weaver, Proc. of SPIE Vol. 7626, 762603 - ( 2010 SPIE

CCC code: $1605-7422 / 10 / \$ 18 \cdot$ doi: $10.1117 / 12.844123$

Proc. of SPIE Vol. $7626762603-1$ 
The aim of this work is to develop and implement a method for establishing a detailed 3D relation between in vivo MRI tumor data and histological sections. The following features needs to be taken into account:

- The large and complex deformations between in vivo MRI and histological sections.

- The artefacts due to the manual steps during histological processing, e.g. orientation differences, staining variations, non-tissue noise, damaged and missing sections.

The key features of our methodology are a very dense histologic sampling, up to 120 histology slices per tumor are acquired, the exploitation of intermediate ex vivo MRI, and a mutual information based non rigid B-spline registration based on whole 3D datasets.

\section{METHODS}

\subsection{Study design}

The study design consists of a number of independent imaging processes as shown in Fig. 1-A. First the rats were inoculated subcutaneously with CA20948 tumor and, when the tumor reached approximately $10 \mathrm{~mm}$ in diameter, imaged using a clinical 3T MRI scanner and a standard clinical wrist coil. After completing the MR examination, animals were sacrificed, and the tumors were gently dissected. During the dissection, the adjacent tumor surfaces were dyed using four tissue dyes in order to track its in vivo orientation. Subsequently, tumors were chemically fixed followed by slicing off a small portion along the longest tumor axis, and embedded in agar solution to prevent tumor movement during ex vivo MRI scanning. The plane for slicing histological sections and a reference plane for ex vivo MRI is defined as follows. The knowledge of the reference plane within in vivo MRI was used to resample in vivo data (shown on the top of Fig.1A.) resembling the ex vivo MRI orientation. Additionally, the reference plane limits the degrees of freedom during separate registration steps. Following the ex vivo MR examination, tumors were processed in histokinette, and subsequently embedded in paraffin from which $4 \mu \mathrm{m}$ sections were sliced and stained. Additionally, the stained sections were digitized at 20x magnification. The digital histology sections were rigidly registered afterwards to the adjacent section and stacked to a 3D set assuming the section thickness to be equal to the distance between the sections, in this case $60 \mu \mathrm{m}$.
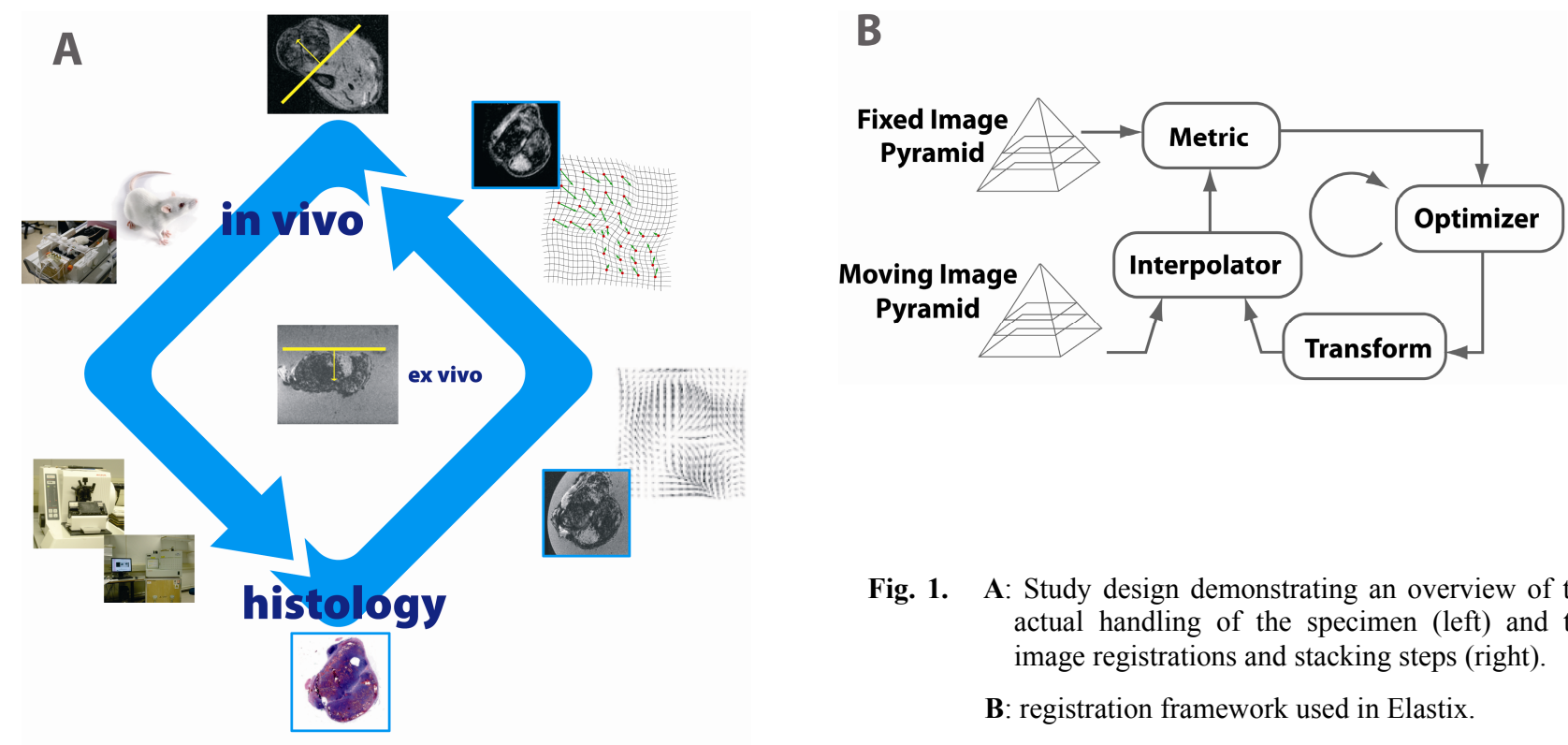

Fig. 1. A: Study design demonstrating an overview of the actual handling of the specimen (left) and the image registrations and stacking steps (right).

B: registration framework used in Elastix. 
The registration consists of two steps: A) registration of 3D in vivo MRI to 3D ex vivo MRI, and B) registration of 3D stacked histology to 3D ex vivo MRI. All registration steps and the concatenation of the acquired transformations were performed using Elastix (http://elastix.isi.uu.nl/) ${ }^{8}$ which is implemented using the publicly available Insight Tool Kit $(\text { ITK })^{9-10}$ see also Fig. 1-B. To account for non-rigid deformations, a high-dimensional deformation model was used for all registrations with the displacement fields parameterized using B-spline models. Automatic 3D registrations were performed by maximization of the mutual information measure with a stochastic gradient descent algorithm ${ }^{11}$.

\subsection{Animal and tumor model}

Lewis rats (Harlan-CPB, Austerlitz, The Netherlands) weighing 250-300g were inoculated subcutaneously in the right hind limb just above the ankle with human pancreatic tumor cells (CA20948 tumor). The animals were inspected daily for tumor growth and general appearance. The tumors were imaged when they reached approximately $10 \mathrm{~mm}$ in diameter. Before the MR imaging, the animals were anesthetized by administration of Hypnorm (Janssen Pharmaceutica, Beerse, Belgium). Analgesia was achieved by intraperitoneal injection of ketamine (Alfasan, Woerden, The Netherlands). During the imaging, the animals were kept at a temperature of $38-39^{\circ} \mathrm{C}$ with a warm water mattress. After completing the MR examination, animals were sacrificed by subcutaneous injection of ketamine, and the tumors were gently dissected. The study was conducted with the approval of the Committee for Animal Research at our institution. In this paper the tumor of one rat is used.

\subsection{Magnetic resonance imaging}

Magnetic resonance imaging was performed using a clinical 3T MRI scanner (Signa Excite, GE Healthcare, USA). A standard clinical wrist coil (Flick Engineering Solutions B.V, The Netherlands) with an internal diameter of $5 \mathrm{~cm}$ was used for both in vivo MRI and ex vivo MRI acquisitions. The 3D susceptibility weighted (T2 $\left.{ }^{*} \mathrm{~W}\right)$ images were used in the registration process because of the excellent structural detail of the tumor content, and a good signal to noise and contrast to noise ratio. In vivo MRI settings: $\mathrm{Tr} / \mathrm{Te}=23.18 / 8.88 \mathrm{~ms}$, flip angle of $10^{\circ}$, and XYZ- resolution of $0.098 \times 0.098 \times 0.2$ $\mathrm{mm}$, and ex vivo MRI settings: $\mathrm{Tr} / \mathrm{Te}=42.17 / 20.88 \mathrm{~ms}$, flip angle of $15^{\circ}$, and XYZ- resolution of $0.059 \times 0.059 \times 0.1$ $\mathrm{mm}$.

\subsection{Specimen processing}

During the dissection, adjacent tumors planes were dyed using four different tissue colours to define the origin of the tumor and track its orientation with the respect to in vivo position. The upper side (underneath the skin), two adjacent sides, and tail side were marked consistently. Immediately after dissection, tumors were placed in $200 \mathrm{cc} 10 \%$ buffered formalin (Boom, The Netherlands). After the fixation process was completed, a thin section of the whole tumor volume was subsequently sliced off along the longest tumor axis in order to define the plane for histological sectioning and thereby create a reference plane for ex vivo MRI, see Fig 2 . The specimen was then gently washed and transferred into a small test tube on a 1\% agar solved in Phosphase Buffered Saline (PBS, AbD Serotec, MorphoSys, Munich, Germany) with the cutted plane down and subsequently embedded into agar to prevent tumor movement and to provide a water rich environment for ex vivo MRI. After the ex vivo MR examination, tumors placed in histokinette for $24 \mathrm{~h}$ processing, and embedded in paraffin, from which two axial slices of $4 \mu \mathrm{m}$ were slices at $60 \mu \mathrm{m}$ intervals using a microtome. Serial sections were mounted on glass slide and stained with hematoxylin and eosin. The position of the individual slices was consequently recorded in a logbook. The slides were digitized using the NanoZoomer Digital Pathology (C9600, Hamamatsu, Japan) at 20x magnification. For registration purposes RGB images are converted to grayscale by eliminating the hue and saturation information while retaining the luminance. 


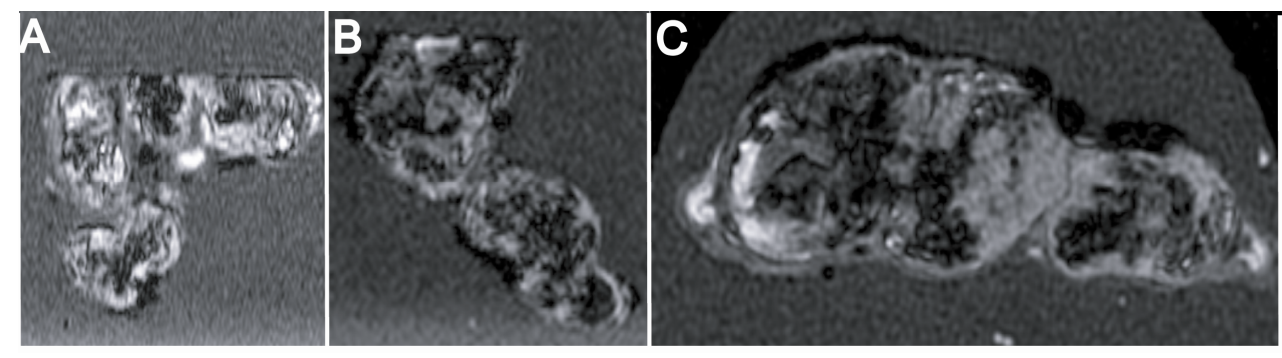

Fig. 2. An example of ex vivo MRI of dissected specimen showing coronal (A), sagittal (B), and axial (C) slices.

\subsection{Registration}

The overall goal is to register in vivo MRI images of the whole tumor to its histological sections. The study design in Fig. 1-A (with on the right hand side registration flow) shows a generalized registration approach. To achieve the goal, the registration is performed using an intermediate ex vivo MRI.

We perform registration as an optimization process that searches for an image transform allowing the closest similarity between consecutive images. The input is a pair of fixed $(F)$ and moving $(M)$ images. The framework iteratively passes $M$ through 4 stages: transform, metric, optimizer, and interpolator as shown in Fig. 1-B. At a given iteration, the transform stage applies transform $T_{n}$ on $M$ which is then resampled onto a grid in the interpolator stage to yield $T_{n}(M)$. The metric stage computes the goodness of fit between $F$ and $T_{n}(M)$ based on the similarity metric values from current and prior iterations allowing the optimizer to refine transform $T_{n+1}$.

Prior to the registrations 2D histology sections were first rigidly registered to its adjacent section, and then stacked to a 3D set. The global transformation consists of two distinct parts: A) registration of 3D ex vivo MRI to 3D in vivo MRI (referred to as ex2in), and B) registration of stacked 2D histological tissue sections to 3D ex vivo MRI (referred to as st2ex). The separate transformations were subsequently concatenated in order to accomplish coregistration of in vivo MRI to histological sections, referred to as st2in. The displacement field was parameterized with a B-spline model with mutual information of a 32-bin histogram similarity measure. Optimisation was performed using adaptive stochastic gradient descent algorithm (11). The registration steps are described in more detail below.

Stacking. Prior to 3D registrations the gray scale images of histological sections were stacked in a 3D data set. Owing the manual character of the process, sections were mounted in different orientations on glass slides. Therefore, in plane rigid registration was needed for each pair of histological sections before stacking. The registration was carried out using a multi resolution approach with a five level Gaussian image pyramid $(16,8,4,2,1)$ for both the fixed and moving image. At the last level, the original images are used for registration. The number of iterations per section was set to 1000 , and computation time per section varied between $60 \mathrm{~s}$ and $70 \mathrm{~s}$. The obtained rigid transformations were then applied to the separate image channels, and the rgb-stack was constructed using MeVisLab application (MeVis Research $\mathrm{GmbH}$, Bremen, Germany) with the adjustment of the slice thickness to $60 \mu \mathrm{m}$ which resembles the actual distance between the sections.

Registration of histology stack to ex vivo MRI (st2ex). Starting from the defined surface (as shown in Fig. 2) in ex vivo MRI, and carefully tracking the progress of the tissue sectioning facilitates registration of 3D stacked histology to ex vivo $3 \mathrm{D}-\mathrm{T} 2{ }^{*} \mathrm{~W}$ MR image. The registration started as rigid, followed by an affine transformation allowing isotropic scaling to account for shrinkage, and finalized by Bspline registration. All registration steps were carried out using a multi resolution approach with a four level Gaussian image pyramid $(8,4,2,1)$ for both fixed (ex vivo 3D-T2 ${ }^{*} \mathrm{w}$ MRI) and moving (3D histology stack) images. The final grid spacing was specified to $4 \mathrm{~mm}$ in all dimensions. The moving image derivative in Z-direction was set to 0 in order to favour the in plane registration and therefore enable managing distortions due to the manual processing of the histological sections. The number of iterations was set to 500, and computation time of the separate registration steps was: $145 \mathrm{~s}, 127 \mathrm{~s}$ and $367 \mathrm{~s}$, respectively.

Registration of ex vivo MRI to in vivo MRI (ex2in). The second stage in the registration process was to perform the coregistration of ex vivo 3D-T2 ${ }^{*} \mathrm{w}$ image and in vivo $3 \mathrm{D}-\mathrm{T} 2{ }^{*} \mathrm{w}$ image. The registration started as rigid, followed by affine allowing isotropic scaling to account for shrinkage, and finally by an elastic registration using a multi resolution approach with a recursive Gaussian image pyramid of four $(8,4,2,1)$, for both fixed (3D in vivo MRI) and moving (3D- 
$\mathrm{T} 2{ }^{*} \mathrm{w}$ ex vivo MRI) image. The final grid spacing was specified to $4 \mathrm{~mm}$ in all dimensions. The number of iterations was set to 500, and computation time of the separate registration steps was: $152 \mathrm{~s}, 145 \mathrm{~s}$ and $392 \mathrm{~s}$, respectively.

\subsection{Experiments}

As a first step, the accuracy of both registrations steps were visually inspected for all slices. To study the volume changes due to the affine and elastic transformations, the determinant of the affine transformation and the determinant of the Jacobian operator of the elastic transformations are computed for the ex 2 in and st2ex transformations separately. To evaluate the effect of registration, a volume of interest (VOI) representing ground-truth viable and necrotic cancerous regions were segmented in the 3D histology stack. Automatic segmentations were generated by transforming the manual segmentations from the histology images to the ex vivo domain and consecutively from the ex vivo domain to the in vivo domain using the transformations computed by the two registration steps. The distribution of the intensities in both transformed VOI's were compared with the distribution of the intensities when only a rigid transformation was applied. As a last step, a segmentation was performed based on the joint histogram distribution of the co-registered ex vivo MRI and 3D stacked histology and the joint histogram distribution of the in vivo MRI and ex vivo MRI and the correspondence with the ground-truth in histology was evaluated.

\section{RESULTS}

Images illustrating both registration steps (st2ex and ex2in) as well as the concatenation of those registrations (st2in) are shown in Figure 3. The first column shows the st2ex registration, the second column shows ex2in, and the third column shows the concatenated st 2 in registration. The registrations were visually inspected using a checker board approach as shown in the last row of Figure 3. The alignment of the tumor borders and of internal structures was evaluated as fairly accurate.

The overall volume preservation was estimated by the determinant of affine part of the ex 2 in and st2ex registrations. It turns out that a significant decrease in volume was observed during ex 2 in registration between ex vivo $3 \mathrm{D}-\mathrm{T} 2{ }^{*} \mathrm{~W}$ MRI and in vivo $3 \mathrm{D}-\mathrm{T} 2{ }^{*} \mathrm{~W}$ MRI, on average $20.6 \%$. This is the inverted effect of the expansion of the tumor after excision from the surrounding tissue, in2ex. Quite the opposite, an increase of the tumor volume was observed during st2ex registration between $3 \mathrm{D}$ histology stack and ex vivo $3 \mathrm{D}-\mathrm{T} 2{ }^{*} \mathrm{w}$ on average $26.9 \%$. This corresponds to the inverted effect of dehydration of the specimen (ex2st). This provides an overall increase of tumor volume of $6.3 \%$. To characterise the properties of the elastic deformation, the Jacobian operator is applied to the separate transformations (ex2in and st2ex), and its determinant is used to estimate the volume change. Table 1 summarizes the results of the elastic transformation. Concisely, although elastic transformation in ex2in preserves the volume while elastic transformation in st2ex show small shrinkage (on average), there is unambiguous difference in the behaviour of the different volume of interest (VOI). Moreover, st2ex transformation has a larger range in the Jacobian determinant, as can be observed in Table 1.

Table 1. Results of the elastic registration showing the range (and mean) of the Jacobian determinant for both transformations.

\begin{tabular}{l|lll} 
registration & Whole tumor & Viable VOI & Necrotic VOI \\
\hline ex2in & $1.00(0.99-1.03)$ & $1.00(0.99-1.02)$ & $1.01(1.00-1.01)$ \\
st2ex & $0.97(0.50-1.00)$ & $0.95(0.50-1.00)$ & $0.89(0.50-1.00$
\end{tabular}

To evaluate the effect of registration, a volume of interest (VOI) representing ground-truth viable and necrotic cancerous regions were annotated in the 3D histology stack. In figure 4, the distributions of ex vivo MRI (first column) and in vivo MRI (second column) intensities segmented by the warped ground-truth volumes of interest (VOI) after rigid (first row) and B-spline (second row) registration. It can clearly be seen, that due to B-spline registration the 'viable VOI' intensity distribution shifts from Gaussian mixture to a more single Gaussian distribution. 

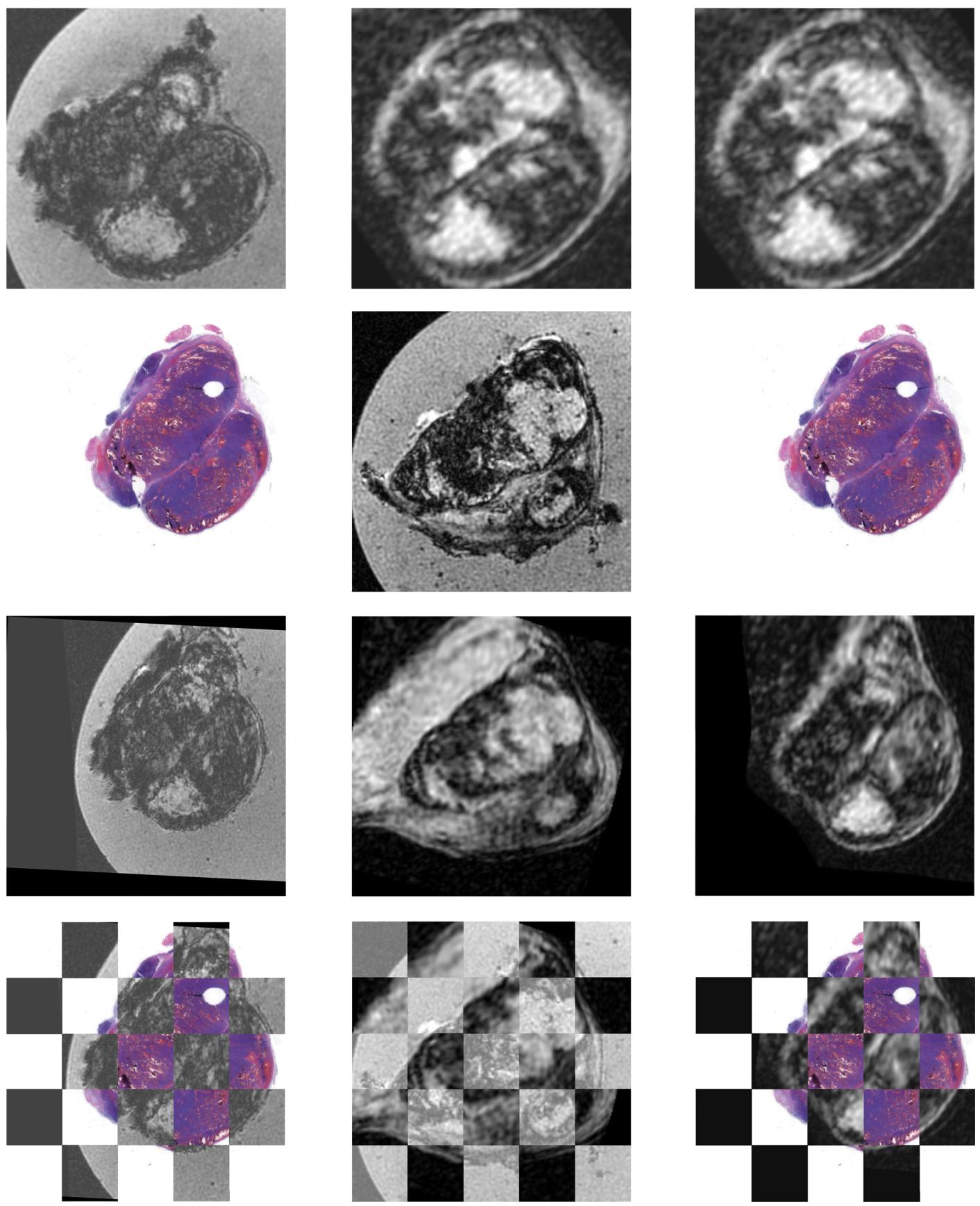

Fig. 3. Illustration of the registration process showing intermediate registration results (column one - st2ex and column two - ex2in), and the whole concatenated st2in registration (third column). First row: Fixed images. Second row: Moving images. Third row: Moving images in register with the fixed images. Forth row: Checkerboard view of corresponding fixed image (first row) and registered moving image (third row). 

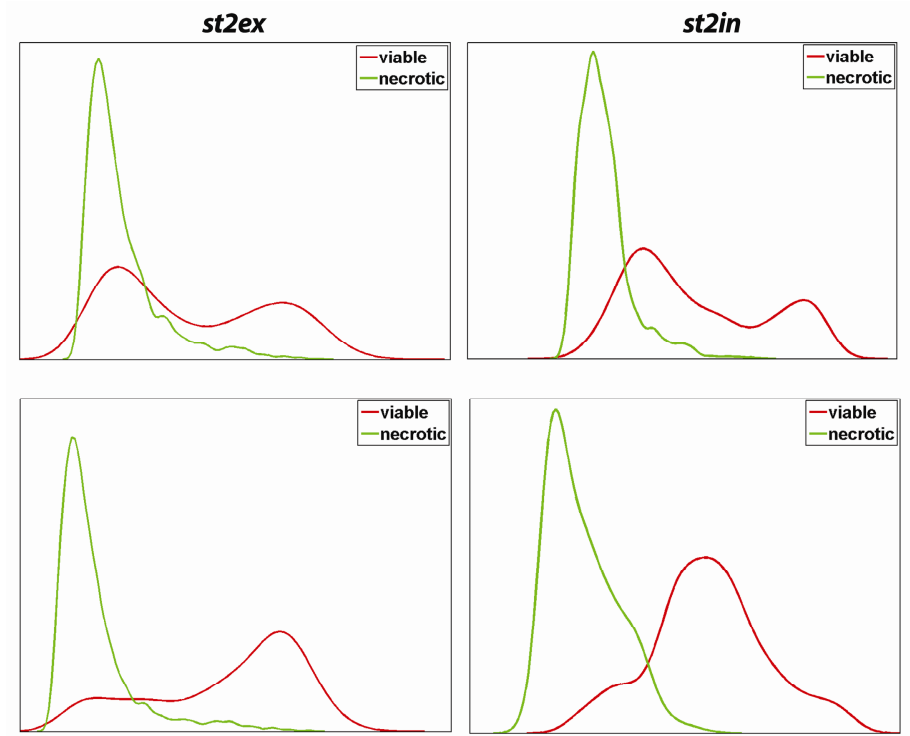

Figure 4. The distributions of ex vivo MRI (first column) and in vivo MRI (second column) intensities segmented by the warped ground-truth volumes of interest (VOI) after rigid (first row) and B-spline (second row) registration. VOIs were annotated in the histological sections. The necrotic VOI is shown in green, while the viable VOI is shown in red.

Figure 5 shows the co-registered ex vivo 3D-T2 ${ }^{*} \mathrm{w}$ MRI (first column) and 3D stacked histology (second column). The third column shows he joint histogram distribution with horizontally the ex vivo intensities and vertically the histology gray-intensities. It can be seen that the gray-histology image is not suitable for the discrimination between viable and other tissue: both tissue types show similar gray-values, whereas in the ex vivo image viable tissue show higher intensity values than not viable tissue.

Figure 6 shows the co-registered in vivo 3D-T2 ${ }^{*} \mathrm{w}$ MRI (first column) and ex vivo 3D-T2 ${ }^{*} \mathrm{w}$ MRI (second column). The third column shows the joint histogram distribution with horizontally the in vivo intensities and vertically the ex vivo gray-intensities. The correspondence between the intensity values of the in vivo and ex vivo MRI can clearly be observed.
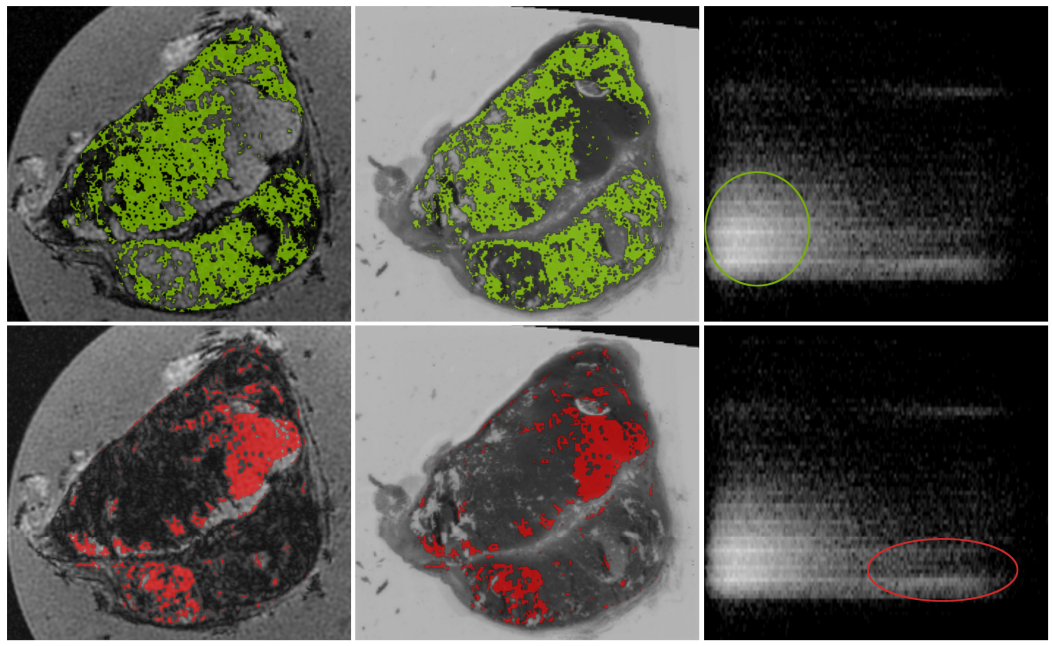

Fig. 5. The joint histogram distribution (third column) of co-registered ex vivo 3D-T2*W MRI (first column-fixed image) and 3D stacked histology (second column), referred to as st2ex. Green/red overlay in the images corresponds to the ellipses in the joint histogram distribution matching with not viable (green) and viable (red) tissue. 


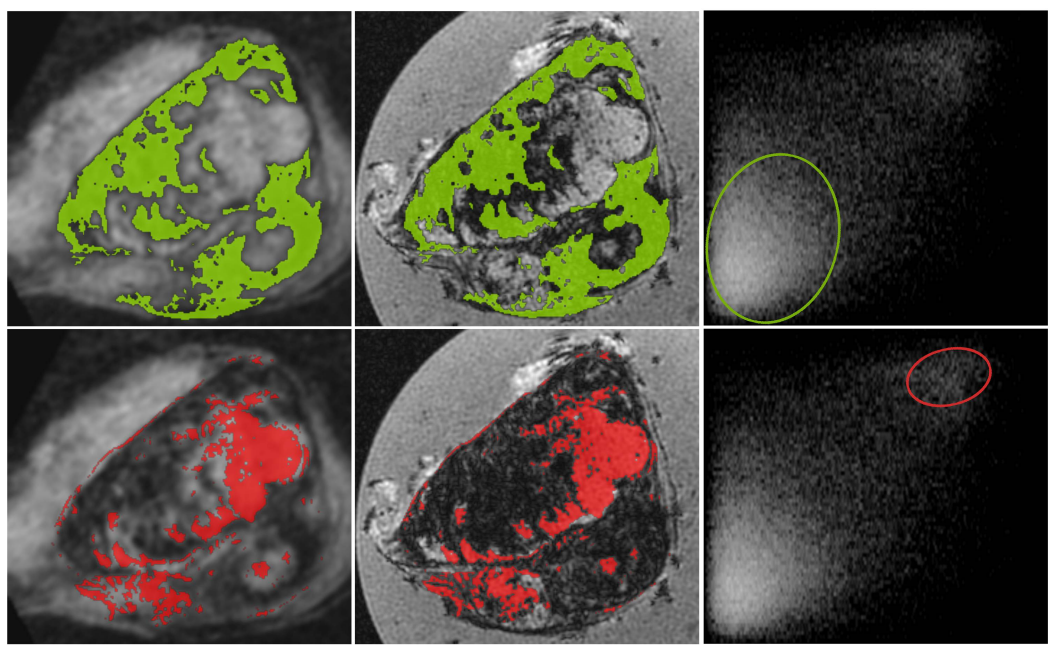

Fig. 6. The joint histogram distribution (third column) of co-registered in vivo 3D-T2*W MRI (first column-fixed image) and ex vivo 3D-T2*W MRI (second column), referred to as ex $2 \mathrm{in}$. Green/red overlay in the images corresponds to the ellipses in the joint histogram distribution matching with not viable (green) and viable (red) tissue.

\section{DISCUSSION AND FUTURE DIRECTIONS}

Non-rigid registration is essential in the process of correlating in vivo MRI data with histology. Matching of histological tissues sections to MR images of tumors is facilitated by using a multi-step registration process involving an ex vivo MRI scan of the tumor. Using this intermediate step, it is possible to account for changes caused by the excision of the tumor, the process of chemical fixation and the histological slicing.

Wang et al. (3) already showed connection between histology and MRI by verifying global tissue traits. Our preliminary results suggest that local registration between in vivo MRI as imaged by $3 \mathrm{D}-\mathrm{T} 2^{*} \mathrm{~W}$ and histological sections is feasible when using a very dense histological sampling, a mutual information based non-rigid B-spline registration and the exploitation of an intermediate ex vivo MRI.

However, a more extensive study is needed in order to validate the registrations. Moreover, there are several possible modifications envisioned which should be explored because they can further increase the robustness and accuracy of the technique without significant increasing process time. For example, using the full colour range of the histology images could help automatic segmentation. Acquiring images with a stereotactic marker system ${ }^{12}$ could facilitate the rigid registration of images. This will speed up the registrations and make manual annotations easier. The use of contrast agent in order to alter the tumor structural detail should be pursued as well.

We have proposed a methodology by which histological annotation can be related back to the corresponding in vivo MRI. This work is of crucial importance to build-up a database of multi-spectral MRI images and multi stained 3D histology which will serve as an essential and valuable source for understanding MR images and the feasibility of MRI to depict biological processes.

\section{PUBLICATION ELSEWHERE}

The work presented in this paper is not presented or published earlier. 


\section{ACKNOWLEDGMENTS}

This work was funded by NWO - Mozaiëk (grant no. 017.002.019), Dutch Cancer Society - KWF (grant no. 20084037), and Erasmus MC seat grant. The authors would also like to thank department of pathology for providing the equipment and assistance in microtome sectioning and staining, and for using their virtual microscope located in the Erasmus MC, Rotterdam, The Netherlands.

\section{REFERENCES}

[1] Jacobs, M.A., Windham, J.P., Soltanian-Zadeh, H., Peck, D.J. and Knight, R.A., "Registration and warping of magnetic resonance images to histological sections", Med Phys, 26(8), 1568-1578 (1999).

[2] Schormann, T. and Zilles, K., "Three-dimensional linear and nonlinear transformations: an integration of light microscopical and MRI data", Hum Brain Mapp, 6(5-6), 339-347 (1998).

[3] Wang, H., Sun, X., Chen, F., De Keyzer, F., Yu, J., Landuyt, W., Vandecaveye, V., Peeters, R., Bosmans, H., Hermans, R., Marchal, G. and Ni, Y., "Treatment of rodent liver tumor with combretastatin a4 phosphate: noninvasive therapeutic evaluation using multiparametric magnetic resonance imaging in correlation with microangiography and histology", Invest Radiol, 44(1), 44-53 (2009).

[4] Jacobs, M.A., Knight, R.A., Soltanian-Zadeh, H., Zheng, Z.G., Goussev, A.V., Peck, D.J., Windham, J.P. and Chopp, M., "Unsupervised segmentation of multiparameter MRI in experimental cerebral ischemia with comparison to T2, diffusion, and ADC MRI parameters and histopathological validation", J Magn Reson Imaging, 11(4), $425-437$ (2000).

[5] Zhan, Y., Ou, Y., Feldman, M., Tomaszeweski, J., Davatzikos, C. and Shen, D., "Registering histologic and MR images of prostate for image-based cancer detection", Acad Radiol, 14(11), 1367-1381 (2007).

[6] Meyer, C.R., Moffat, B.A., Kuszpit, K.K., Bland, P.L., Mckeever, P.E., Johnson, T.D., Chenevert, T.L., Rehemtulla, A. and Ross, B.D., "A methodology for registration of a histological slide and in vivo MRI volume based on optimizing mutual information", Mol Imaging, 5(1), 16-23 (2006).

[7] Park, H., Piert, M.R., Khan, A., Shah, R., Hussain, H., Siddiqui, J., Chenevert, T.L. and Meyer, C.R., "Registration methodology for histological sections and in vivo imaging of human prostate", Acad Radiol, 15(8), 1027-1039 (2008).

[8] Klein, S., Staring, M., Murphy, K., Viergever, M.A. and Pluim, J.P., "Elastix: a toolbox for intensity-based medical image registration", IEEE transactions on medical imaging, 29(1), 196-205 (2010).

[9] Ibanez L, Schroeder W, Ng L, Cates J. "The ITK Software Guide", Kitware Inc. (2004).

[10] Yoo, T.S., Ackerman, M.J., Lorensen, W.E., Schroeder, W., Chalana, V., Aylward, S., Metaxas, D. and Whitaker, R., "Engineering and algorithm design for an image processing Api: a technical report on ITK--the Insight Toolkit", Stud Health Technol Inform, 85, 586-592 (2002).

[11] Rueckert,D., Sonoda, L.I., Hayes, C., Hill, D.L., Leach, M.O. and Hawkes, D.J., "Nonrigid registration using free-form deformations: application to breast MR images", IEEE Trans Med Imaging, 18(8), 712-721 (1999).

[12] Humm, J.L., Ballon, D., Hu, Y.C., Ruan, S., Chui, C., Tulipano, P.K., Erdi, A., Koutcher, J., Zakian, K., Urano, M., Zanzonico, P., Mattis, C., Dyke, J., Chen, Y., Harrington, P., O'Donoghue, J.A. nad Ling, C.C., "A stereotactic method for the three-dimensional registration of multi-modality biologic images in animals: NMR, PET, histology, and autoradiography", Medical physics 30(9), 2303-2314 (2003). 